\title{
1 High-resolution Mapping of Forest Carbon Stocks in the Colombian Amazon
}

2 Supplementary Material

3

4

5

(I) Field validation

A summary of the allometric equations used, and species encountered is provided in Tables S1 and S2, respectively.

\section{(II) Model fitting techniques}

The ACD validation model (eq. 3) and H-D model (Table S1) were each fit using non-linear Maximum Likelihood Analysis in $\mathrm{R}$, and by incorporating a third equation parameter in the form of a non-arithmetic error term (i.e., $y=a x^{b}+x^{k} * \varepsilon$ ) to account for heteroscedasticity common to previously published $\mathrm{ACD}-\mathrm{MCH}$ and $\mathrm{H}-\mathrm{D}$ relationships. This fitting technique is analogous to fitting a linear model to log-transformed $x$ and $y$ data, thereby avoiding the need for logtransformation and back-transformation (Baskerville, 1972; Mascaro et al., 2011b).

\section{(III) Additional information about the regional stratification approach}

For the final regional stratification, a total of 147 Landsat Thematic Mapper (TM) and Enhanced Thematic Mapper Plus (ETM+) images were processed through CLASlite. This dataset included 72 images for 2010, 31 for 1990, 20 for 2000, and 24 for 2005. Employing a semi-automated approach to masking clouds, smoke, haze and shadows, we constructed cloud-free regional maps at $30 \mathrm{~m}$ resolution.

Median ACD values determined from LiDAR-scale ACD for each land-cover class are listed in Table S3. If a class in the final stratification map did not contain sufficient LiDAR coverage (e.g., less than the targeted $1 \%$ or less than $100 \mathrm{ha}$ ), we assigned the median ACD value of a broader class on the preceding node of the stratification decision tree (Figure $2 \mathrm{c}$ of main text). For example, if the high TRI stratum for a particular catchment did not have sufficient coverage, we assigned the median ACD value of the same TRI stratum for all catchments.

For the stratification approach, catchment boundaries were derived from SRTM data (Hydrology Toolbox, ArcGIS 10, ESRI Inc., USA), which resulted in additional ACD substratification by more than $60 \mathrm{Mg} \mathrm{Cha}^{-1}$ in some cases. This addition - supported by a stratification approach - allowed for capturing discrete geographic changes in carbon stocks that may otherwise have been missed in the regional analyses, similar to the localized effects of terrain variation as expressed in the TRI. 
Following the results of the correlation analyses (see Methods of main text), we conducted multiple least squares regression analyses using elevation (ELEV), slope, aspect and a terrain ruggedness index (TRI) derived from NASA Shuttle Radar Topography Mission (SRTM) data, and photosynthetic vegetation coverage fraction (PV) and soil cover fraction derived from CLASlite. Through iterations of regression analyses using all of these variables, as well as their interaction terms, we determined that only PV and ELEV influenced the fit of the model at the scale of the entire study region. The final least squares regression of these variables yielded the following model:

$$
A C D=-3553.5097-0.1691 \times E L E V+79.9664 \times P V-0.4323 \times P V^{2}
$$

with an adjusted $r^{2}$ of 0.20 and a residual standard error of $33.85 \mathrm{Mg} \mathrm{C}^{-1}$. All terms were significant at $P<0.0001$. This equation, which ingested all available LiDAR data was used estimate $A C D$ at the regional level.

While the above model was used for final mapping (i.e., in conjunction with non-forest carbon stock estimations from CLASlite), we also created a second model using a $75 \%$ subset of the LiDAR data for the purposes of estimating errors in the remaining $25 \%$ of the data. This model was:

$$
A C D=-3669.4719-0.1672 \times E L E V+82.5010 \times P V-0.4462 \times P V^{2}
$$

with an adjusted $r^{2}$ of 0.19 and a residual standard error of $34.19 \mathrm{Mg} \mathrm{C}^{-1}$. All terms were significant at $P<0.0001$.

\section{(V) Sources of error in linking LiDAR-based and plot-based estimates of ACD}

Standard plot protocol in the field (e.g., Condit, 1998) dictates that trees are considered to be inside a plot if more than $50 \%$ of their main stem is contained within the plot boundary. However, LiDAR energy is returned by the 3-D components of forest canopies, including tree crowns and branches. Thus, a plot within the LiDAR coverage includes portions of crowns that overhang the edge of the plot, and excludes portions that extent beyond the plot edge. In effect, this disagreement between LiDAR and field estimated ACD produces a false error in typical LiDAR calibrations, including those of the type underlying the universal LiDAR model. Plot size also has a considerable effect, with errors decreasing as plot size increases according to the inverse square root of the plot area.

Mascaro et al. (2011a) demonstrated the propagation of each of these errors using a 50-ha plot with mapped trees. Empirically, relative errors were $23 \mathrm{Mg} \mathrm{Cha}^{-1}$ at 0.09 ha resolution (i.e., a median value based on measurements at 0.08 and 0.1 ha resolution), or $21.5 \%$ of the mean 
63 carbon stock of the entire 50-ha area $\left(107 \mathrm{Mg} \mathrm{Cha}^{-1}\right)$. Likewise, errors were $10.7 \mathrm{Mg} \mathrm{C}^{-1}$ at 1 64 ha resolution (10.0\%).

65 
Asner, G. P., Powell, G. V. N., Mascaro, J., Knapp, D. E., Clark, J. K., Jacobson, J., KennedyBowdoin, T., Balaji, A., Paez-Acosta, G., Victoria, E., Secada, L., Valqui, M., and Hughes, R.F.: High-resolution forest carbon stocks and emissions in the Amazon, P. Natl. A. Sci., 107, 16738-16742, 2010.

Baskerville, G.: Use of logarithmic regression in the estimation of plant biomass, Canadian Journal of Forest Research-Revue Canadienne De Recherche Forestiere, 2, 49-53, 1972.

Chao, K.-J., Phillips, O. L., Baker, T. R., Peacock, J., Lopez-Gonzalez, G., Vásquez Martínez, R., Monteagudo, A., and Torres-Lezama, A.: After trees die: Quantities and determinants of necromass across amazonia, Biogeosciences, 6, 1615-1626, 2009.

Chave, J., Andalo, C., Brown, S., Cairns, M. A., Chambers, J. Q., Eamus, D., Fölster, H., Fromard, F., Higuchi, N., Puig, H., Riéra, B., and Yamakura, T.: Tree allometry and improved estimation of carbon stocks and balance in tropical forests, Oecologia, 145, 87-99, DOI 10.1007/s00442-005-0100-x, 2005.

Chave, J., Coomes, D., Jansen, S., Lewis, S. L., Swenson, N. G., and Zanne, A. E.: Towards a worldwide wood economics spectrum, Ecology Letters, 12, 351-366, 2009.

Condit, R.: Tropical forest census plots, Springer-Verlag and R. G. Landes Company, Berlin, Germany and Georgetown, Texas, USA, 1998.

Mascaro, J., Detto, M., Asner, G. P., and Muller-Landau, H. C.: Evaluating uncertainty in mapping forest carbon with airborne LiDAR, Remote Sens. Environ., 115, 3770-3774, 2011a.

Mascaro, J., Litton, C. M., Hughes, F. R., Uowolo, A., and Schnitzer, S. A.: Minimizing bias in biomass allometry: Model selection and log-transformation of data, Biotropica, 43, 649653, 2011b.

Riley, S. J., DeGloria, S. D., and Elliot, R.: A terrain ruggedness index that quantifies topographic heterogeneity, Intermountain Journal of Science, 5, 23-27, 1999.

ter Steege, H., Pitman, N. C. A., Phillips, O. L., Chave, J., Sabatier, D., Duque, A., Molino, J. F., Prevost, M. F., Spichiger, R., Castellanos, H., von Hildebrand, P., and Vasquez, R.: Continental-scale patterns of canopy tree composition and function across amazonia, Nature, 443, 444-447, 10.1038/nature05134, 2006. 
(a)

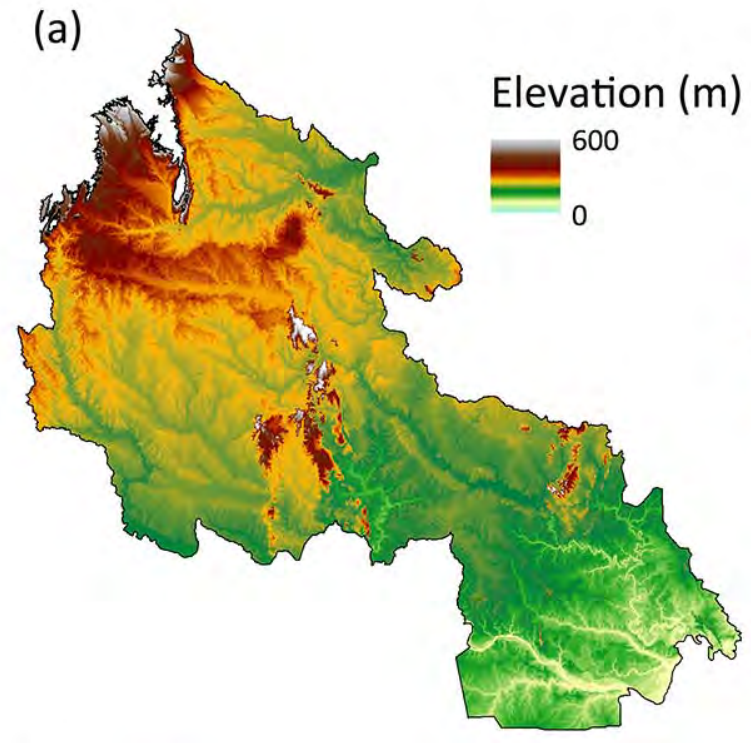

(c)

(c)

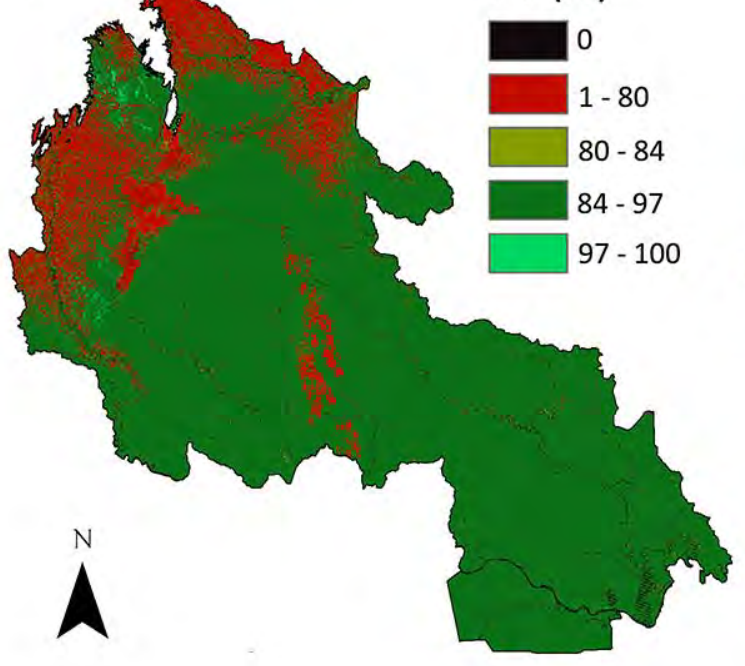

(b)

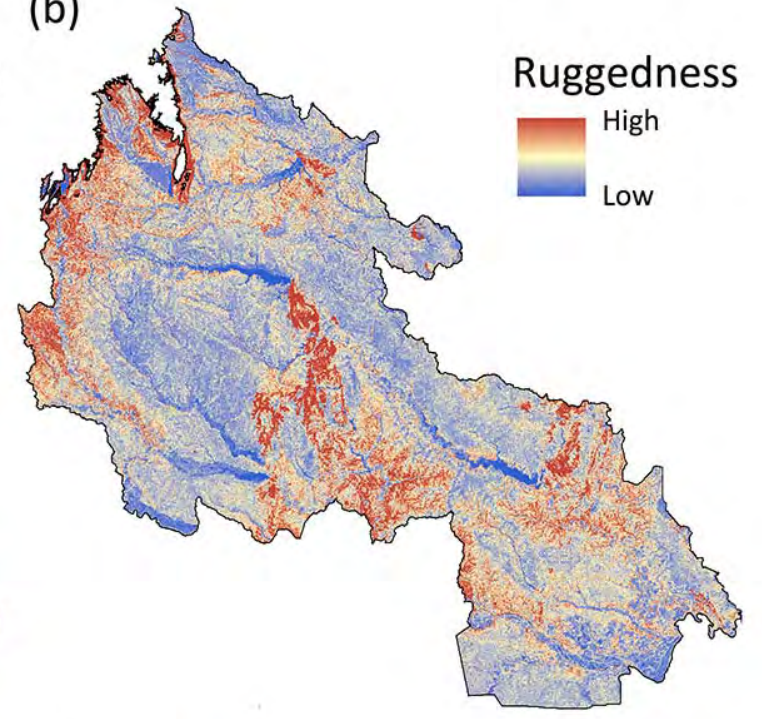

(d)

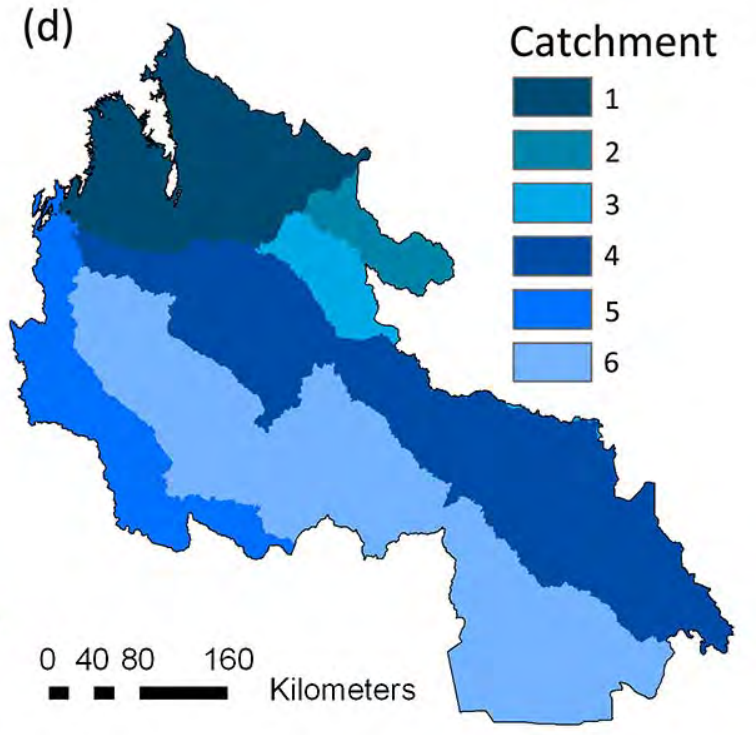

98

99

100

101

102

103

Figure S1. Selected variables used to stratify the study area: (a) digital elevation model (DEM) derived from the NASA Shuttle Radar Topography Mission (STRM), (b) terrain ruggedness index (TRI), (c) fractional cover of photosynthetic vegetation (PV) derived from CLASlite, and (d) drainage catchments. Elevation and PV were also used as inputs to the regression approach. 

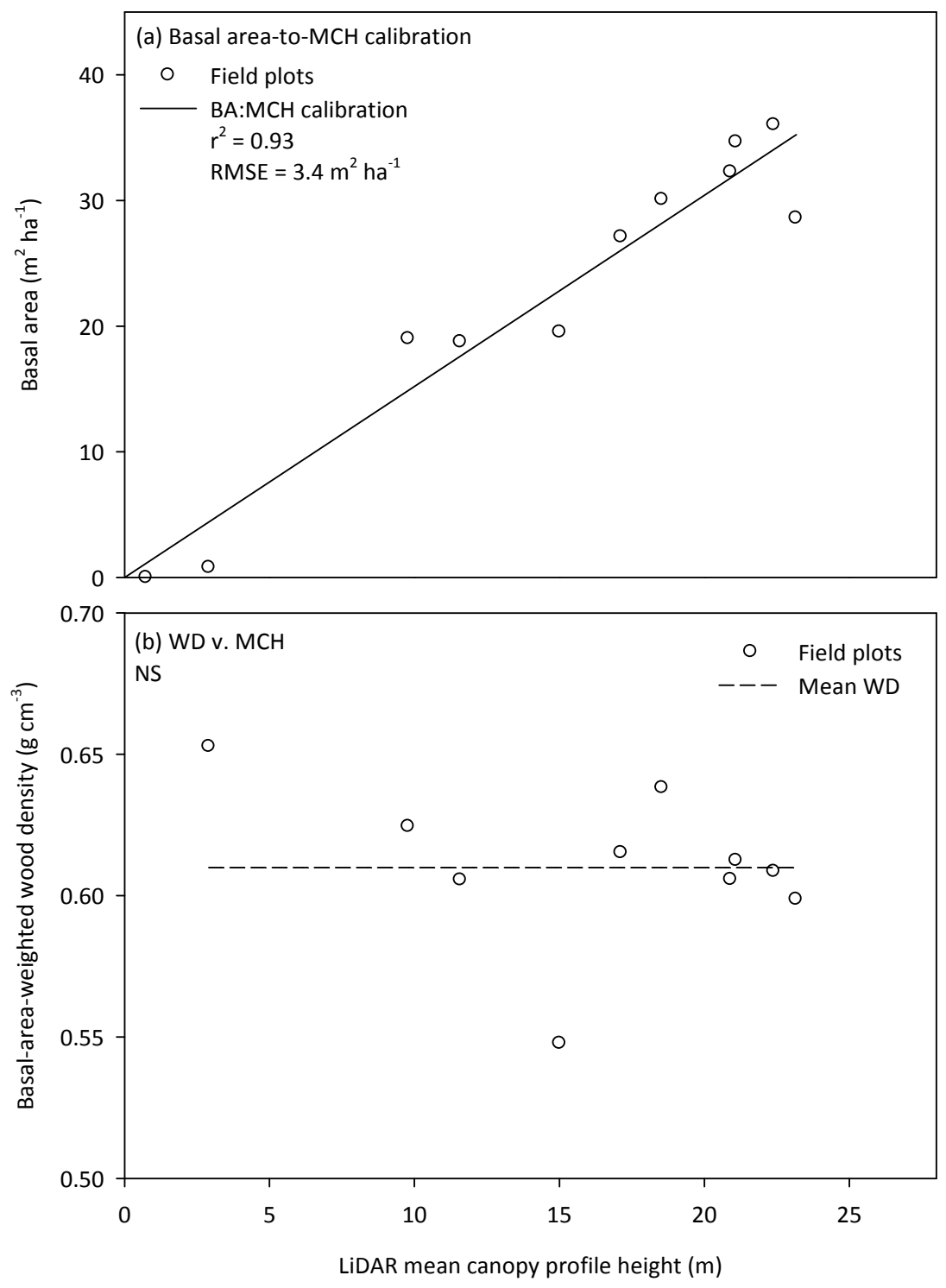

104

105

106 Figure S2. (a) Relationship between field-estimated basal area and LiDAR-derived mean canopy 107 profile height (termed the Stocking Coefficient). (b) Relationship between basal-area-weighted 108 wood density and LiDAR derived mean canopy profile height.

109

110

111

112 


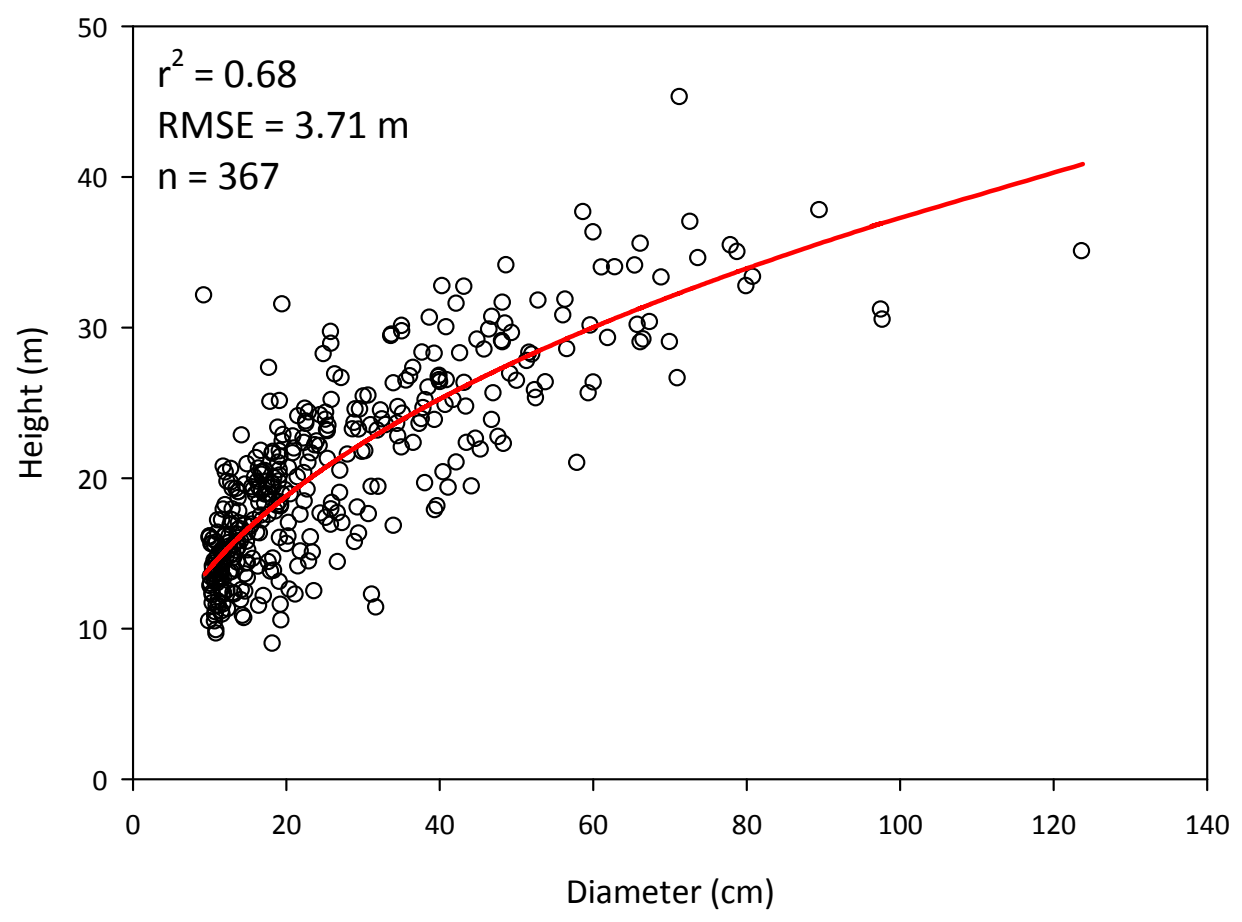

117 Figure S3. Height to diameter relationship among 367 trees measured in the field plots. All 118 trees $>50 \mathrm{~cm}$ dbh were measured for height, as well as several trees of smaller diameters. For 119 field ACD validation, heights of the remaining smaller trees were estimated using the model as 120 shown (model parameters in Table S1).

121 

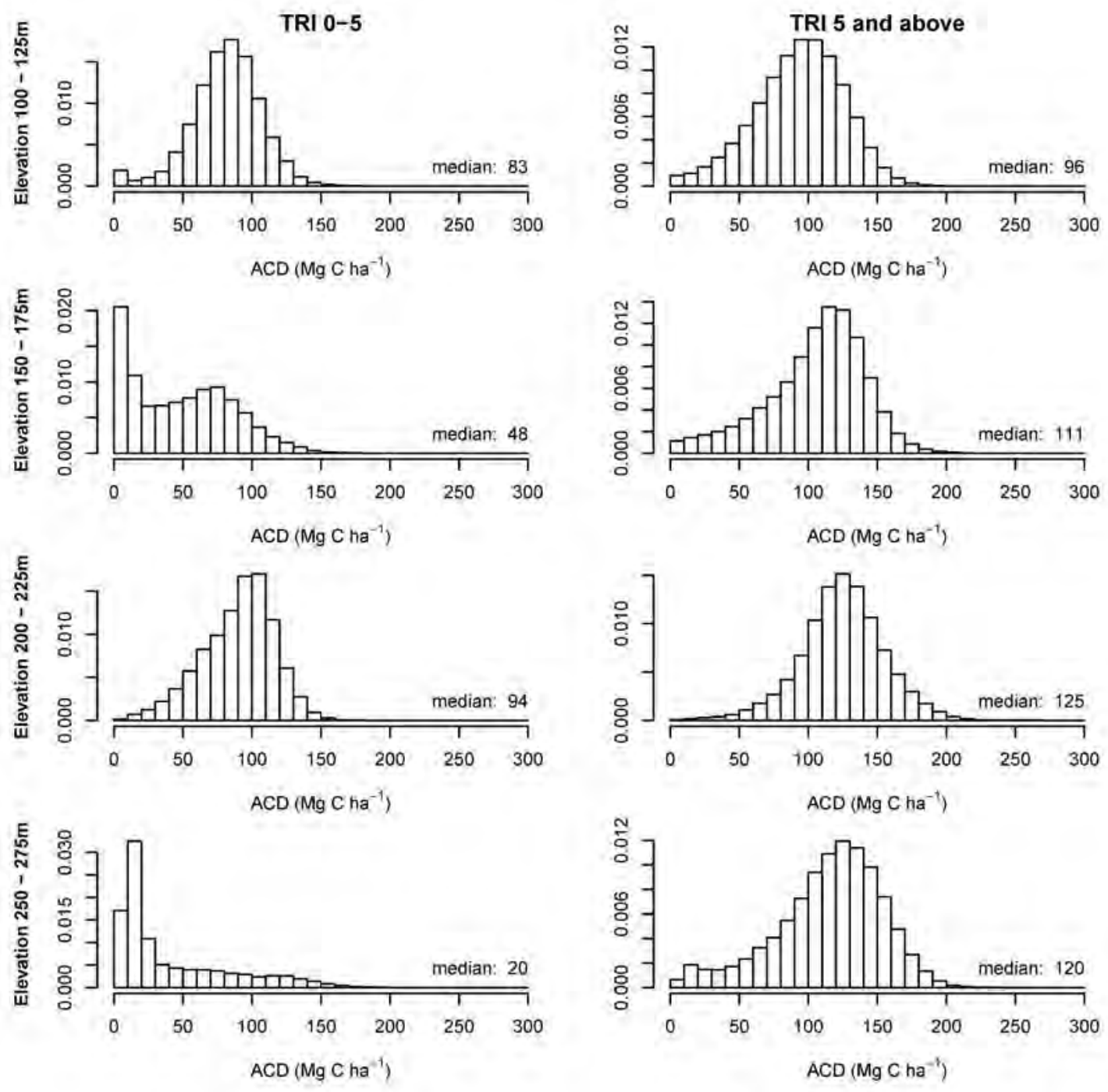

124

125 Figure S4. Differences in the distribution of forest aboveground carbon density (ACD) at 126 different elevations for landscapes with low (0-5) and high (5+) values of the terrain ruggedness 127 index (TRI; Riley et al., 1999). Median ACD values for each distribution are shown in the lower 128 right of each panel. 


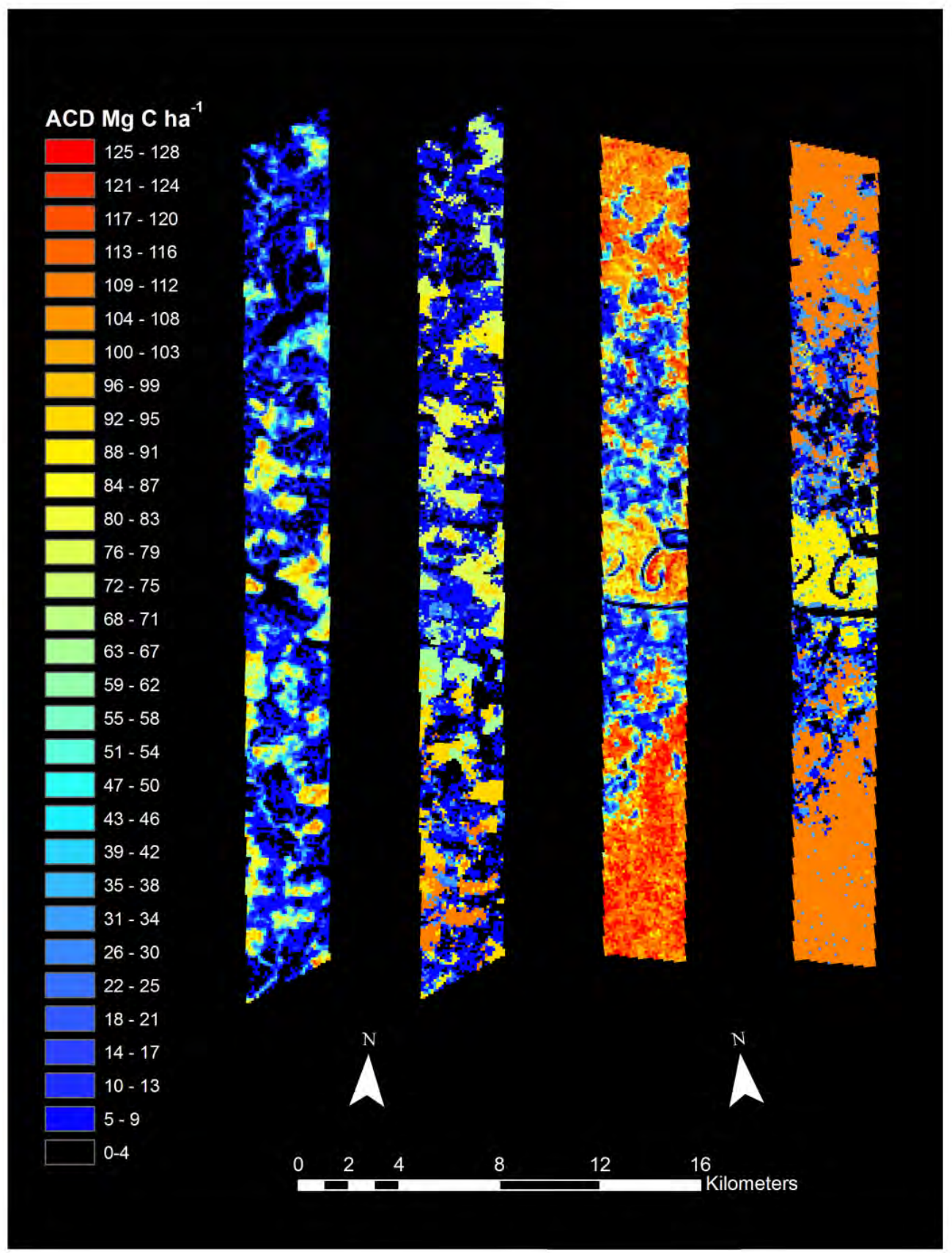

132 Figure S5. Side-by-side comparison of LiDAR-based ACD (left) and regionally-mapped ACD 133 (right) according to the stratification approach. 


\section{Stratification Approach}

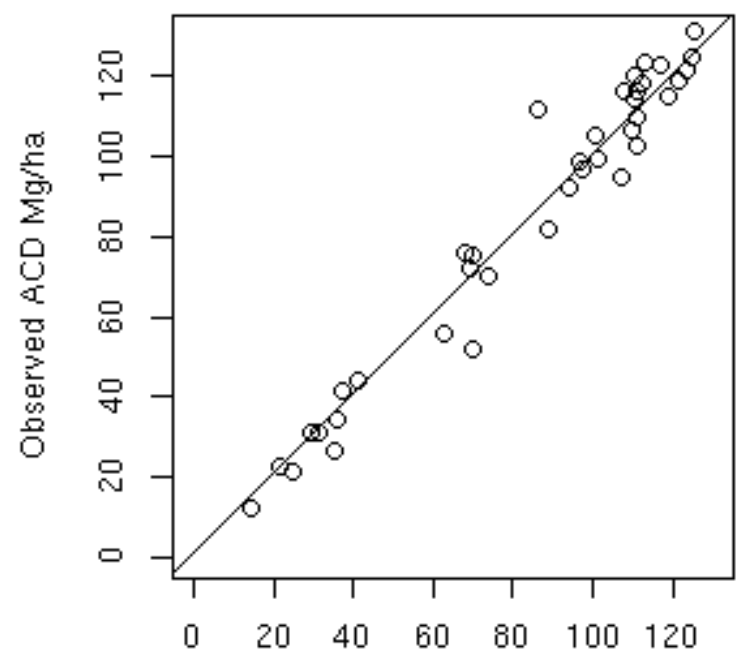

Predicted ACD Mgiha

\section{Regression Approach}

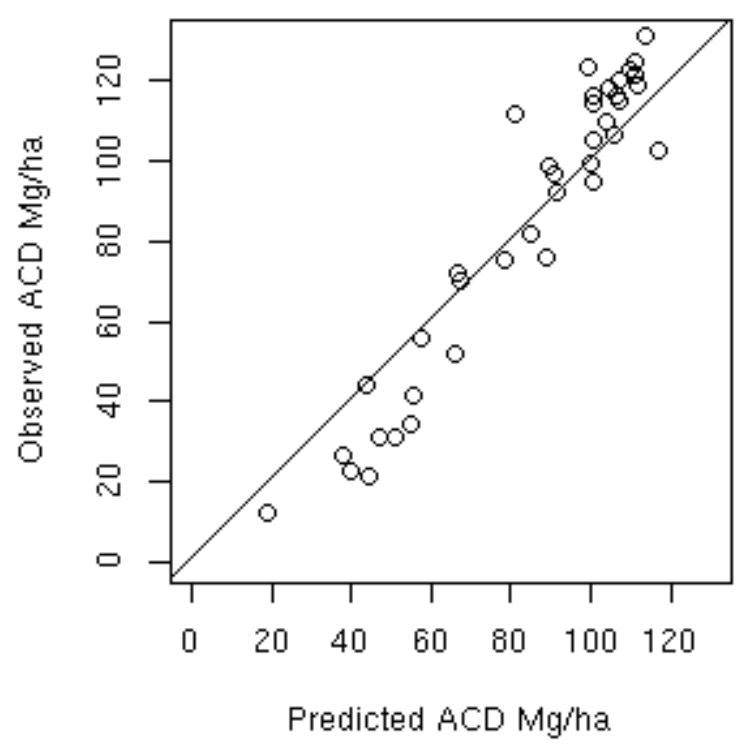

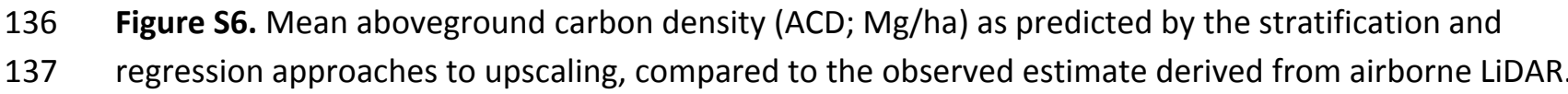
138 Each point represents the validation region of one of 38 flight polygons. These areas comprise the $25 \%$ 139 of the LiDAR coverage remaining after excluding a $2600 \mathrm{~m}$ strip down the center of each polygon that 140 was used to train the upscaling models. The line depicts a 1:1 relationship. 
141 Table S1. Allometric models used to estimate aboveground biomass (AGB; $\mathrm{kg}$ ) or height (H; $\mathrm{m}$ ).

142 Diameters $(D, c m)$ were measured at breast height (1.3 $\mathrm{m}$ from the base) or above buttress.

143 Wood densities $\left(\rho ; \mathrm{g} \mathrm{cm}^{-3}\right)$ were taken from a global wood density database (Chave et al., 2009)

144 based on species-, genus-, or family-level identification (see Table S2).

\begin{tabular}{llcl}
\hline Parameter & Equation & $\mathbf{r}^{2}$ & Reference \\
\hline AGB & $0.0776^{*}\left(D^{2 *} H^{*} \rho\right)^{0.94}$ & $0.96^{1}$ & Chave et al. (2005) \\
H & $5.2497^{*} D^{0.4258}$ & 0.68 & This study \\
AGB & $\pi^{*}\left(0.5^{*} D\right)^{2 *} \rho^{*} H / 10$ & $\mathrm{n} / \mathrm{a}^{2}$ & Asner et al. (2010) \\
AGB & $\pi^{*}\left(0.5^{*} \mathrm{D}\right)^{2 *}\left(1.17^{*} \rho-0.21\right) * \mathrm{H} / 10^{*} 0.5$ & $\mathrm{n} / \mathrm{a}^{2}$ & Asner et al. (2010) \\
\hline
\end{tabular}

${ }^{1}$ coefficient of determination for $\ln (\mathrm{y})$

${ }^{2}$ Palm and dead tree biomass was estimated using the formula for volume of a cylinder, corrected for wood density, and in the case of dead trees for trunk taper and decay typical of standing dead Amazonian trees (Chao et al., 2009). 
147 Table S2. Summary of all stems encountered in the field validation plots. Wood densities were 148 taken from Chave et al. (2009) according to either family or genus-level identification. When a 149 value was not available from Chave et al. (2009), a default regional value of 0.58 was applied 150 (ter Steege et al., 2006).

\begin{tabular}{|c|c|c|c|c|c|c|}
\hline Family & Genus & Species & $\begin{array}{l}\text { Number } \\
\text { of Stems }\end{array}$ & $\begin{array}{l}\text { Wood Density } \\
\qquad\left(\mathrm{g} \mathrm{cm}^{-3}\right)\end{array}$ & $\begin{array}{c}\text { WD } \\
\text { Determination }\end{array}$ & Reference \\
\hline ANACARDIACEAE & & & 2 & 0.56 & Family & Chave \\
\hline ANACARDIACEAE & Tapirira & spp. & 6 & 0.37 & Genus & Chave \\
\hline ANNONACEAE & & & 37 & 0.56 & Family & Chave \\
\hline ANNONACEAE & Duguetia & spp. & 1 & 0.73 & Genus & Chave \\
\hline ANNONACEAE & Xylopia & spp. & 36 & 0.59 & Genus & Chave \\
\hline APOCYNACEAE & & & 33 & 0.57 & Family & Chave \\
\hline APOCYNACEAE & Aspidosperma & spp. & 15 & 0.75 & Genus & Chave \\
\hline APOCYNACEAE & Couma & macrocarpa & 1 & 0.53 & Genus & Chave \\
\hline APOCYNACEAE & Couma & spp. & 3 & 0.53 & Genus & Chave \\
\hline APOCYNACEAE & Hymatanthus & spp. & 1 & 0.55 & Genus & Chave \\
\hline ARALIACEAE & Schefflera & spp. & 2 & 0.39 & Genus & Chave \\
\hline ARECACEAE & & & 1 & 0.56 & Family & Chave \\
\hline ARECACEAE & Astrocaryum & spp. & 1 & 0.51 & Genus & Chave \\
\hline ARECACEAE & Euterpe & precatoria & 9 & 0.39 & Genus & Chave \\
\hline ARECACEAE & Iriartea & spp. & 1 & 0.27 & Genus & Chave \\
\hline ARECACEAE & Mauritiella & spp. & 8 & 0.58 & Region & Default \\
\hline ARECACEAE & Oenocarpus & bacaba & 1 & 0.68 & Genus & Chave \\
\hline ARECACEAE & Oenocarpus & bataua & 45 & 0.68 & Genus & Chave \\
\hline ARECACEAE & Oenocarpus & spp. & 10 & 0.68 & Genus & Chave \\
\hline ARECACEAE & Socratea & exhorriza & 2 & 0.23 & Genus & Chave \\
\hline BIGNONIACEAE & Callychlamys & spp. & 1 & 0.58 & Region & Default \\
\hline BIXACEAE & Bixa & spp. & 2 & 0.35 & Genus & Chave \\
\hline BOMBACACEAE & & & 9 & 0.48 & Family & Chave \\
\hline BOMBACACEAE & Pachira & spp. & 2 & 0.48 & Genus & Chave \\
\hline BOMBACACEAE & Scleronema & spp. & 9 & 0.61 & Genus & Chave \\
\hline BURSERACEAE & & & 21 & 0.52 & Family & Chave \\
\hline BURSERACEAE & Dacryodes & spp. & 1 & 0.57 & Genus & Chave \\
\hline BURSERACEAE & Protium & spp. & 8 & 0.57 & Genus & Chave \\
\hline CAESALPINIACEAE & & & 36 & 0.68 & Family & Chave \\
\hline CAESALPINIACEAE & Hymenaea & spp. & 1 & 0.80 & Genus & Chave \\
\hline CAESALPINIACEAE & Macrolobium & spp. & 19 & 0.62 & Genus & Chave \\
\hline CAESALPINIACEAE & Tachigali & spp. & 16 & 0.58 & Genus & Chave \\
\hline CARYOCARIACEAE & & & 1 & 0.70 & Family & Chave \\
\hline CARYOCARIACEAE & Caryocar & spp. & 4 & 0.69 & Genus & Chave \\
\hline CECROPIACEAE & & & 4 & 0.34 & Family & Chave \\
\hline
\end{tabular}




\begin{tabular}{|c|c|c|c|c|c|c|}
\hline Family & Genus & Species & $\begin{array}{l}\text { Number } \\
\text { of Stems }\end{array}$ & $\begin{array}{l}\text { Wood Density } \\
\qquad\left(\mathrm{g} \mathrm{cm}^{-3}\right)\end{array}$ & $\begin{array}{c}\text { WD } \\
\text { Determination }\end{array}$ & Reference \\
\hline CECROPIACEAE & Cecropia & spp. & 2 & 0.34 & Genus & Chave \\
\hline CECROPIACEAE & Pourouma & spp. & 13 & 0.39 & Genus & Chave \\
\hline CELASTRACEAE & & & 5 & 0.66 & Family & Chave \\
\hline CELASTRACEAE & Goupia & glabra & 2 & 0.73 & Genus & Chave \\
\hline CELASTRACEAE & Goupia & spp. & 1 & 0.73 & Genus & Chave \\
\hline CELASTRACEAE & Lagupia & spp. & 1 & 0.58 & Region & Default \\
\hline CHRYSOBALANACEAE & & & 67 & 0.78 & Family & Chave \\
\hline CHRYSOBALANACEAE & Licania & spp. & 4 & 0.82 & Genus & Chave \\
\hline CLUSIACEAE & & & 8 & 0.65 & Family & Chave \\
\hline CLUSIACEAE & Caraipa & spp. & 4 & 0.66 & Genus & Chave \\
\hline CLUSIACEAE & Clusia & spp. & 3 & 0.68 & Genus & Chave \\
\hline CLUSIACEAE & Tovomita & spp. & 7 & 0.70 & Genus & Chave \\
\hline CLUSIACEAE & Vismia & spp. & 3 & 0.49 & Genus & Chave \\
\hline COMBRETACEAE & & & 1 & 0.60 & Family & Chave \\
\hline COMBRETACEAE & Buchenavia & spp. & 4 & 0.75 & Genus & Chave \\
\hline COMBRETACEAE & Conceveiba & spp. & 1 & 0.41 & Genus & Chave \\
\hline EBENACEAE & Dyospiros & spp. & 1 & 0.68 & Genus & Chave \\
\hline ELAEOCARPACEAE & & & 5 & 0.55 & Family & Chave \\
\hline ELAEOCARPACEAE & Sloanea & spp. & 3 & 0.61 & Genus & Chave \\
\hline EUPHORBIACEAE & & & 10 & 0.51 & Family & Chave \\
\hline EUPHORBIACEAE & Conceveiba & spp. & 7 & 0.41 & Genus & Chave \\
\hline EUPHORBIACEAE & Hevea & spp. & 73 & 0.48 & Genus & Chave \\
\hline EUPHORBIACEAE & Mabea & spp. & 14 & 0.61 & Genus & Chave \\
\hline EUPHORBIACEAE & Senefeldera & spp. & 9 & 0.78 & Genus & Chave \\
\hline FABACEAE & & & 45 & 0.68 & Family & Chave \\
\hline FABACEAE & Clatrotropis & macrocarpa & 1 & 0.79 & Genus & Chave \\
\hline FABACEAE & Clatrotropis & spp. & 75 & 0.79 & Genus & Chave \\
\hline FABACEAE & Ormosia & spp. & 2 & 0.58 & Genus & Chave \\
\hline FABACEAE & Swartzia & spp. & 1 & 0.85 & Genus & Chave \\
\hline HUMIRIACEAE & & & 8 & 0.77 & Family & Chave \\
\hline ICACINACEAE & & & 12 & 0.58 & Family & Chave \\
\hline LAURACEAE & & & 50 & 0.56 & Family & Chave \\
\hline LAURACEAE & Ocotea & spp. & 5 & 0.54 & Genus & Chave \\
\hline LECYTHIDACEAE & & & 113 & 0.70 & Family & Chave \\
\hline LECYTHIDACEAE & Eschweilera & spp. & 12 & 0.83 & Genus & Chave \\
\hline MELASTOMATACEAE & & & 3 & 0.67 & Family & Chave \\
\hline MELASTOMATACEAE & Miconia & spp. & 1 & 0.63 & Genus & Chave \\
\hline MIMOSACEAE & & & 5 & 0.68 & Family & Chave \\
\hline MIMOSACEAE & Inga & spp. & 13 & 0.58 & Genus & Chave \\
\hline MIMOSACEAE & Parkia & spp. & 17 & 0.46 & Genus & Chave \\
\hline
\end{tabular}




\begin{tabular}{|c|c|c|c|c|c|c|}
\hline Family & Genus & Species & $\begin{array}{l}\text { Number } \\
\text { of Stems }\end{array}$ & $\begin{array}{l}\text { Wood Density } \\
\left(\mathrm{g} \mathrm{cm}^{-3}\right)\end{array}$ & $\begin{array}{c}\text { WD } \\
\text { Determination }\end{array}$ & Reference \\
\hline MIMOSACEAE & Zygia & spp. & 16 & 0.82 & Genus & Chave \\
\hline MORACEAE & & & 65 & 0.54 & Family & Chave \\
\hline MORACEAE & Ficus & spp. & 1 & 0.41 & Genus & Chave \\
\hline MORACEAE & Pseudolmedia & spp. & 2 & 0.67 & Genus & Chave \\
\hline MYRISTICACEAE & & & 33 & 0.51 & Family & Chave \\
\hline MYRISTICACEAE & Iryanthera & spp. & 30 & 0.59 & Genus & Chave \\
\hline MYRISTICACEAE & Virola & spp. & 34 & 0.48 & Genus & Chave \\
\hline MYRTACEAE & & & 33 & 0.77 & Family & Chave \\
\hline NYCTAGINACEAE & & & 2 & 0.52 & Family & Chave \\
\hline OLACACEAE & & & 3 & 0.76 & Family & Chave \\
\hline OLACACEAE & Minquartia & spp. & 4 & 0.79 & Genus & Chave \\
\hline POLYGONACEAE & Coccoloba & spp. & 2 & 0.69 & Genus & Chave \\
\hline RUBIACEAE & & & 56 & 0.64 & Family & Chave \\
\hline SAPINDACEAE & & & 6 & 0.69 & Family & Chave \\
\hline SAPINDACEAE & Allophyllus & spp. & 4 & 0.52 & Genus & Chave \\
\hline SAPINDACEAE & Cupania & spp. & 2 & 0.61 & Genus & Chave \\
\hline SAPOTACEAE & & & 151 & 0.70 & Family & Chave \\
\hline SAPOTACEAE & Manilkara & spp. & 3 & 0.89 & Genus & Chave \\
\hline SAPOTACEAE & Micropholis & spp. & 20 & 0.66 & Genus & Chave \\
\hline SAPOTACEAE & Pouteria & spp. & 6 & 0.69 & Genus & Chave \\
\hline STERCULIACEAE & & & 5 & 0.48 & Family & Chave \\
\hline STERCULIACEAE & Sterculia & spp. & 5 & 0.40 & Genus & Chave \\
\hline STERCULIACEAE & Theobroma & spp. & 4 & 0.53 & Genus & Chave \\
\hline TILIACEAE & & & 14 & 0.48 & Family & Chave \\
\hline TILIACEAE & Apeiba & spp. & 24 & 0.25 & Genus & Chave \\
\hline TILIACEAE & Mollia & spp. & 12 & 0.49 & Genus & Chave \\
\hline VERBENACEAE & Vitex & spp. & 1 & 0.57 & Genus & Chave \\
\hline VIOLACEAE & & & 4 & 0.65 & Family & Chave \\
\hline VIOLACEAE & Rinorea & spp. & 1 & 0.68 & Genus & Chave \\
\hline VOCHYSIACEAE & & & 19 & 0.55 & Family & Chave \\
\hline VOCHYSIACEAE & Erisma & spp. & 42 & 0.57 & Genus & Chave \\
\hline VOCHYSIACEAE & Qualea & spp. & 3 & 0.65 & Genus & Chave \\
\hline VOCHYSIACEAE & Vochysia & spp. & 2 & 0.49 & Genus & Chave \\
\hline Unknown & & & 499 & 0.58 & Region & Default \\
\hline Total stems & & & 2068 & & & \\
\hline \multicolumn{2}{|c|}{ Unweighted mean WD } & & & 0.59 & & \\
\hline \multicolumn{2}{|c|}{ \% Family determination } & & & & 41.92 & \\
\hline \multicolumn{2}{|c|}{$\%$ Genus determination } & & & & 33.46 & \\
\hline \multicolumn{2}{|c|}{$\%$ Region determination } & & & & 24.61 & \\
\hline
\end{tabular}


151 Table S3. Summary of vegetation classes used in final regional stratification. Elevation (m) a.s.I. 152 (ELEV), fractional cover (\%) of photosynthetic vegetation (PV), terrain ruggedness index (TRI), 153 and catchment (CM) are shown. Mean, median, and standard deviation of LiDAR-based 154 aboveground carbon density (ACD) are given in $\mathrm{Mg} \mathrm{C} \mathrm{ha-1.} \mathrm{Total} \mathrm{extent} \mathrm{of} \mathrm{LiDAR} \mathrm{coverage} \mathrm{(ha)}$ 155 and total extent of each class in the regional ACD map (ha) are provided, as well as the relative 156 coverage of LiDAR (\%) within each class. An asterisk (*) indicates that ACD statistics were 157 calculated from a broader class due to $<100$ ha of LiDAR coverage.

\begin{tabular}{|c|c|c|c|c|c|c|c|c|c|c|c|}
\hline $\begin{array}{c}\text { CLASS } \\
\text { NUMBER }\end{array}$ & & ELEV & PV & TRI & $\mathrm{CM}$ & $\begin{array}{l}\text { Mean } \\
A C D\end{array}$ & $\begin{array}{l}\text { Median } \\
\text { ACD }\end{array}$ & $\begin{array}{l}\text { St. Dev. } \\
\text { ACD }\end{array}$ & $\begin{array}{c}\text { LiDAR } \\
\text { (ha) }\end{array}$ & $\begin{array}{l}\text { Map } \\
\text { (ha) }\end{array}$ & $\begin{array}{c}\text { Cover } \\
(\%)\end{array}$ \\
\hline \multicolumn{12}{|l|}{ Non-forest } \\
\hline 1 & & & $(0,20]$ & & & 3.1 & 0.2 & 8.7 & 944 & 42429 & 2.22 \\
\hline 2 & & & $(20,40]$ & & & 4.0 & 0.4 & 10.8 & 6152 & 226653 & 2.71 \\
\hline 3 & & & $(40,60]$ & & & 4.3 & 0.6 & 10.8 & 22661 & 735553 & 3.08 \\
\hline 4 & & & $(60,80]$ & & & 12.9 & 5.4 & 22.7 & 35437 & 1062902 & 3.33 \\
\hline 5 & & & $(80,100]$ & & & 40.5 & 32.9 & 35.4 & 6296 & 216462 & 2.91 \\
\hline \multicolumn{12}{|c|}{ Deforestation regrowth } \\
\hline 6 & 5 Years & & & & & 29.8 & 21.8 & 28.9 & 582 & 21356 & 2.73 \\
\hline 7 & 10 Years & & & & & 30.4 & 23.1 & 26.5 & 2230 & 57978 & 3.85 \\
\hline \multicolumn{12}{|c|}{ Disturbance regrowth } \\
\hline 8 & All ages & & & & & 53.0 & 46.6 & 38.5 & 1022 & 31506 & 3.24 \\
\hline \multicolumn{12}{|l|}{ Forest } \\
\hline $9 *$ & & $<100$ & {$[80,84)$} & & & 98.6 & 100.3 & 26.6 & 15 & 4314 & 0.35 \\
\hline 10 & & $<100$ & $\geq 97$ & & & 98.4 & 100.7 & 28.5 & 105 & 5339 & 1.96 \\
\hline 11 & & {$[100,125)$} & {$[80,84)$} & & & 74.0 & 73.3 & 37.5 & 113 & 4254 & 2.67 \\
\hline 12 & & {$[100,125)$} & $\geq 97$ & & & 67.2 & 67.3 & 34.1 & 178 & 4896 & 3.64 \\
\hline 13 & & {$[125,150)$} & {$[80,84)$} & & & 85.7 & 86.2 & 36.1 & 914 & 9442 & 9.68 \\
\hline 14 & & {$[125,150)$} & $\geq 97$ & & & 107.7 & 111.5 & 38.6 & 277 & 4864 & 5.69 \\
\hline 15 & & {$[150,175)$} & {$[80,84)$} & & & 87.0 & 91.1 & 43.9 & 487 & 11998 & 4.06 \\
\hline 16 & & {$[150,175)$} & $\geq 97$ & & & 108.4 & 107.0 & 39.3 & 142 & 4291 & 3.31 \\
\hline 17 & & {$[175,200)$} & {$[80,84)$} & & & 48.8 & 39.6 & 39.0 & 1100 & 22577 & 4.87 \\
\hline 18 & & {$[175,200)$} & $\geq 97$ & & & 90.7 & 88.1 & 42.7 & 664 & 17021 & 3.90 \\
\hline 19 & & {$[200,225)$} & {$[80,84)$} & & & 37.0 & 25.0 & 35.0 & 1683 & 43820 & 3.84 \\
\hline $20^{*}$ & & {$[200,225)$} & $\geq 97$ & & & 104.7 & 105.1 & 49.3 & 671 & 36875 & 1.82 \\
\hline 21 & & {$[225,250)$} & {$[80,84)$} & & & 41.4 & 32.6 & 35.4 & 1518 & 44846 & 3.38 \\
\hline 22 & & {$[225,250)$} & $\geq 97$ & & & 90.2 & 86.7 & 30.9 & 1322 & 62927 & 2.10 \\
\hline 23 & & {$[250,275)$} & {$[80,84)$} & & & 41.7 & 31.9 & 35.6 & 849 & 40519 & 2.10 \\
\hline 24 & & {$[250,275)$} & $\geq 97$ & & & 92.0 & 89.8 & 29.4 & 651 & 56393 & 1.15 \\
\hline 25 & & {$[275,300)$} & {$[80,84)$} & & & 41.1 & 29.9 & 35.8 & 587 & 25592 & 2.29 \\
\hline 26 & & {$[275,300)$} & $\geq 97$ & & & 88.2 & 86.8 & 22.5 & 1499 & 31768 & 4.72 \\
\hline 27 & & {$[300,325)$} & {$[80,84)$} & & & 33.1 & 20.0 & 36.5 & 415 & 14345 & 2.90 \\
\hline 28 & & {$[300,325)$} & $\geq 97$ & & & 89.0 & 86.9 & 25.9 & 1384 & 27924 & 4.96 \\
\hline
\end{tabular}




\begin{tabular}{|c|c|c|c|c|c|c|c|c|c|c|}
\hline 29 & {$[325,350)$} & {$[80,84)$} & & & 25.8 & 15.1 & 30.7 & 418 & 9174 & 4.55 \\
\hline 30 & {$[325,350)$} & $\geq 97$ & & & 91.9 & 92.6 & 35.2 & 535 & 24660 & 2.17 \\
\hline 31 & {$[350,375)$} & {$[80,84)$} & & & 31.3 & 19.8 & 32.3 & 268 & 6112 & 4.39 \\
\hline 32 & {$[350,375)$} & $\geq 97$ & & & 82.7 & 81.9 & 37.9 & 349 & 19915 & 1.75 \\
\hline 33 & {$[375,400)$} & {$[80,84)$} & & & 36.6 & 24.2 & 34.4 & 244 & 4576 & 5.33 \\
\hline 34 & {$[375,400)$} & $\geq 97$ & & & 81.9 & 83.3 & 29.3 & 464 & 13962 & 3.32 \\
\hline 35 & {$[400,500)$} & {$[80,84)$} & & & 35.7 & 17.9 & 39.1 & 673 & 9498 & 7.09 \\
\hline 36 & {$[400,500)$} & $\geq 97$ & & & 69.4 & 65.7 & 31.3 & 664 & 27491 & 2.42 \\
\hline 37 & $\geq 500$ & {$[80,84)$} & & & 33.1 & 16.4 & 35.0 & 600 & 8175 & 7.34 \\
\hline 38 & $\geq 500$ & $\geq 97$ & & & 72.4 & 74.6 & 34.5 & 142 & 15284 & 0.93 \\
\hline 39 & $<100$ & {$[84,97)$} & {$[0,5)$} & & 92.5 & 89.2 & 30.6 & 451 & 17746 & 2.54 \\
\hline $40^{*}$ & $<100$ & {$[84,97)$} & $\geq 5$ & $\mathrm{~F}$ & 98.8 & 100.4 & 26.3 & 0 & 53342 & 0.00 \\
\hline 41 & $<100$ & {$[84,97)$} & $\geq 5$ & $\mathrm{H}$ & 98.8 & 100.4 & 26.3 & 2129 & 99273 & 2.14 \\
\hline 42 & {$[100,125)$} & {$[84,97)$} & {$[0,5)$} & & 40.3 & 19.9 & 39.9 & 738 & 27057 & 2.73 \\
\hline 43 & {$[100,125)$} & {$[84,97)$} & $\geq 5$ & $\mathrm{~F}$ & 118.7 & 119.9 & 25.9 & 1051 & 295063 & 0.36 \\
\hline 44 & {$[100,125)$} & {$[84,97)$} & $\geq 5$ & $\mathrm{H}$ & 112.7 & 118.4 & 39.3 & 8159 & 480575 & 1.70 \\
\hline 45 & {$[125,150)$} & {$[84,97)$} & {$[0,5)$} & & 89.5 & 96.1 & 33.9 & 2521 & 22465 & 11.22 \\
\hline 46 & {$[125,150)$} & {$[84,97)$} & $\geq 5$ & $\mathrm{~F}$ & 134.7 & 137.4 & 30.7 & 12033 & 487044 & 2.47 \\
\hline 47 & {$[125,150)$} & {$[84,97)$} & $\geq 5$ & $\mathrm{H}$ & 125.3 & 126.6 & 28.7 & 35978 & 894347 & 4.02 \\
\hline $48^{*}$ & {$[125,150)$} & {$[84,97)$} & $\geq 5$ & I & 127.7 & 129.0 & 29.5 & 0 & 1075 & 0.00 \\
\hline 49 & {$[150,175)$} & {$[84,97)$} & {$[0,5)$} & & 89.1 & 92.6 & 25.5 & 3120 & 64763 & 4.82 \\
\hline $50 *$ & {$[150,175)$} & {$[84,97)$} & $\geq 5$ & C & 124.4 & 124.5 & 29.2 & 0 & 288 & 0.00 \\
\hline 51 & {$[150,175)$} & {$[84,97)$} & $\geq 5$ & D & 67.5 & 70.0 & 19.7 & 308 & 854 & 35.99 \\
\hline 52 & {$[150,175)$} & {$[84,97)$} & $\geq 5$ & $\mathrm{~F}$ & 118.7 & 118.1 & 34.1 & 8334 & 498378 & 1.67 \\
\hline $53^{*}$ & {$[150,175)$} & {$[84,97)$} & $\geq 5$ & G & 124.4 & 124.5 & 29.2 & 0 & 59239 & 0.00 \\
\hline 54 & {$[150,175)$} & {$[84,97)$} & $\geq 5$ & $\mathrm{H}$ & 127.3 & 126.5 & 26.0 & 22558 & 570354 & 3.96 \\
\hline $55^{*}$ & {$[150,175)$} & {$[84,97)$} & $\geq 5$ & I & 124.4 & 124.5 & 29.2 & 0 & 3685 & 0.00 \\
\hline 56 & {$[175,200)$} & {$[84,97)$} & {$[0,5)$} & & 70.4 & 72.5 & 32.0 & 1519 & 28143 & 5.40 \\
\hline 57 & {$[175,200)$} & {$[84,97)$} & $\geq 5$ & C & 54.8 & 55.1 & 26.9 & 6022 & 51134 & 11.78 \\
\hline 58 & {$[175,200)$} & {$[84,97)$} & $\geq 5$ & D & 84.8 & 84.7 & 26.7 & 6124 & 64085 & 9.56 \\
\hline 59 & {$[175,200)$} & {$[84,97)$} & $\geq 5$ & $\mathrm{~F}$ & 111.8 & 112.9 & 29.6 & 20528 & 789486 & 2.60 \\
\hline 60 & {$[175,200)$} & {$[84,97)$} & $\geq 5$ & G & 85.2 & 87.6 & 33.9 & 1151 & 333779 & 0.34 \\
\hline 61 & {$[175,200)$} & {$[84,97)$} & $\geq 5$ & $\mathrm{H}$ & 119.5 & 120.3 & 25.3 & 15989 & 762784 & 2.10 \\
\hline $62^{*}$ & {$[175,200)$} & {$[84,97)$} & $\geq 5$ & I & 103.4 & 107.4 & 34.9 & 0 & 2491 & 0.00 \\
\hline 63 & {$[200,225)$} & {$[84,97)$} & {$[0,5)$} & & 47.9 & 46.5 & 35.9 & 2085 & 108875 & 1.91 \\
\hline 64 & {$[200,225)$} & {$[84,97)$} & $\geq 5$ & C & 46.5 & 44.5 & 28.5 & 4262 & 120186 & 3.55 \\
\hline 65 & {$[200,225)$} & {$[84,97)$} & $\geq 5$ & D & 71.6 & 70.2 & 30.3 & 4185 & 201630 & 2.08 \\
\hline 66 & {$[200,225)$} & {$[84,97)$} & $\geq 5$ & $E$ & 73.0 & 71.9 & 28.1 & 1920 & 42735 & 4.49 \\
\hline 67 & {$[200,225)$} & {$[84,97)$} & $\geq 5$ & $\mathrm{~F}$ & 114.7 & 116.4 & 29.5 & 23211 & 759033 & 3.06 \\
\hline 68 & {$[200,225)$} & {$[84,97)$} & $\geq 5$ & G & 102.8 & 107.2 & 29.7 & 3240 & 395970 & 0.82 \\
\hline 69 & {$[200,225)$} & {$[84,97)$} & $\geq 5$ & $\mathrm{H}$ & 114.2 & 116.6 & 25.6 & 18553 & 794812 & 2.33 \\
\hline $70^{*}$ & {$[200,225)$} & {$[84,97)$} & $\geq 5$ & I & 103.9 & 109.6 & 35.3 & 0 & 898 & 0.00 \\
\hline 71 & {$[225,250)$} & {$[84,97)$} & {$[0,5)$} & & 73.1 & 70.4 & 36.2 & 747 & 30607 & 2.44 \\
\hline
\end{tabular}




\begin{tabular}{|c|c|c|c|c|c|c|c|c|c|c|}
\hline 72 & {$[225,250)$} & {$[84,97)$} & $\geq 5$ & C & 54.5 & 54.8 & 23.6 & 6861 & 293979 & 2.33 \\
\hline 73 & {$[225,250)$} & {$[84,97)$} & $\geq 5$ & D & 56.7 & 55.5 & 28.7 & 1159 & 101518 & 1.14 \\
\hline 74 & {$[225,250)$} & {$[84,97)$} & $\geq 5$ & $E$ & 106.4 & 107.7 & 25.7 & 15949 & 261248 & 6.10 \\
\hline 75 & {$[225,250)$} & {$[84,97)$} & $\geq 5$ & $F$ & 110.3 & 113.0 & 28.0 & 20096 & 667876 & 3.01 \\
\hline 76 & {$[225,250)$} & {$[84,97)$} & $\geq 5$ & G & 105.2 & 109.6 & 31.4 & 2400 & 204874 & 1.17 \\
\hline 77 & {$[225,250)$} & {$[84,97)$} & $\geq 5$ & $\mathrm{H}$ & 109.6 & 112.7 & 27.8 & 21715 & 921188 & 2.36 \\
\hline $78^{*}$ & {$[225,250)$} & {$[84,97)$} & $\geq 5$ & 1 & 102.5 & 107.4 & 32.3 & 0 & $<1$ & 0.00 \\
\hline 79 & {$[250,275)$} & {$[84,97)$} & {$[0,5)$} & & 80.6 & 82.3 & 25.5 & 1807 & 61716 & 2.93 \\
\hline 80 & {$[250,275)$} & {$[84,97)$} & $\geq 5$ & C & 63.2 & 63.6 & 25.2 & 5899 & 288751 & 2.04 \\
\hline 81 & {$[250,275)$} & {$[84,97)$} & $\geq 5$ & D & 75.1 & 75.1 & 49.8 & 173 & 22553 & 0.77 \\
\hline 82 & {$[250,275)$} & {$[84,97)$} & $\geq 5$ & $E$ & 102.2 & 102.1 & 23.4 & 2978 & 129279 & 2.30 \\
\hline 83 & {$[250,275)$} & {$[84,97)$} & $\geq 5$ & $F$ & 98.8 & 99.5 & 27.0 & 13517 & 475777 & 2.84 \\
\hline $84^{*}$ & {$[250,275)$} & {$[84,97)$} & $\geq 5$ & G & 92.6 & 94.9 & 31.8 & 29 & 114640 & 0.02 \\
\hline 85 & {$[250,275)$} & {$[84,97)$} & $\geq 5$ & $\mathrm{H}$ & 101.7 & 106.7 & 33.0 & 6906 & 338265 & 2.04 \\
\hline 86 & {$[275,300)$} & {$[84,97)$} & {$[0,5)$} & & 79.3 & 79.1 & 17.5 & 699 & 6985 & 10.01 \\
\hline 87 & {$[275,300)$} & {$[84,97)$} & $\geq 5$ & C & 74.7 & 76.6 & 24.4 & 5307 & 147247 & 3.60 \\
\hline $88^{*}$ & {$[275,300)$} & {$[84,97)$} & $\geq 5$ & D & 86.3 & 88.1 & 28.0 & 62 & 6110 & 1.02 \\
\hline 89 & {$[275,300)$} & {$[84,97)$} & $\geq 5$ & $E$ & 100.4 & 98.9 & 19.4 & 1083 & 17128 & 6.32 \\
\hline 90 & {$[275,300)$} & {$[84,97)$} & $\geq 5$ & $F$ & 90.1 & 91.8 & 26.4 & 12727 & 340886 & 3.73 \\
\hline $91 *$ & {$[275,300)$} & {$[84,97)$} & $\geq 5$ & G & 86.3 & 88.1 & 28.0 & 0 & 42191 & 0.00 \\
\hline 92 & {$[275,300)$} & {$[84,97)$} & $\geq 5$ & $\mathrm{H}$ & 85.8 & 91.3 & 39.5 & 1650 & 69960 & 2.36 \\
\hline $93^{*}$ & {$[300,325)$} & {$[84,97)$} & {$[0,5)$} & & 86.1 & 90.2 & 34.3 & 82 & 2228 & 3.68 \\
\hline 94 & {$[300,325)$} & {$[84,97)$} & $\geq 5$ & C & 77.9 & 79.1 & 25.7 & 2389 & 87873 & 2.72 \\
\hline 95 & {$[300,325)$} & {$[84,97)$} & $\geq 5$ & D & 66.8 & 40.7 & 74.2 & 102 & 2073 & 4.92 \\
\hline 96 & {$[300,325)$} & {$[84,97)$} & $\geq 5$ & $E$ & 104.3 & 104.2 & 19.5 & 2678 & 21621 & 12.39 \\
\hline 97 & {$[300,325)$} & {$[84,97)$} & $\geq 5$ & $\mathrm{~F}$ & 84.6 & 89.6 & 37.6 & 5607 & 123349 & 4.55 \\
\hline $98^{*}$ & {$[300,325)$} & {$[84,97)$} & $\geq 5$ & G & 86.1 & 90.2 & 34.3 & 0 & 11702 & 0.00 \\
\hline 99 & {$[300,325)$} & {$[84,97)$} & $\geq 5$ & $\mathrm{H}$ & 66.4 & 65.8 & 36.3 & 931 & 26436 & 3.52 \\
\hline $100 *$ & {$[325,350)$} & {$[84,97)$} & {$[0,5)$} & & 76.0 & 77.9 & 40.6 & 28 & 825 & 3.42 \\
\hline 101 & {$[325,350)$} & {$[84,97)$} & $\geq 5$ & C & 58.6 & 56.0 & 37.2 & 534 & 56166 & 0.95 \\
\hline 102 & {$[325,350)$} & {$[84,97)$} & $\geq 5$ & D & 50.4 & 38.7 & 50.5 & 135 & 909 & 14.85 \\
\hline 103 & {$[325,350)$} & {$[84,97)$} & $\geq 5$ & $E$ & 100.7 & 100.9 & 18.6 & 749 & 14654 & 5.11 \\
\hline 104 & {$[325,350)$} & {$[84,97)$} & $\geq 5$ & $\mathrm{~F}$ & 75.7 & 75.3 & 41.3 & 3886 & 39293 & 9.89 \\
\hline $105^{*}$ & {$[325,350)$} & {$[84,97)$} & $\geq 5$ & G & 76.1 & 78.0 & 40.6 & 0 & 6128 & 0.00 \\
\hline 106 & {$[325,350)$} & {$[84,97)$} & $\geq 5$ & $\mathrm{H}$ & 69.3 & 63.5 & 41.4 & 582 & 17649 & 3.30 \\
\hline $107 *$ & {$[350,375)$} & {$[84,97)$} & {$[0,5)$} & & 68.7 & 65.5 & 36.4 & 43 & 294 & 14.62 \\
\hline 108 & {$[350,375)$} & {$[84,97)$} & $\geq 5$ & C & 66.2 & 67.0 & 30.4 & 1500 & 40794 & 3.68 \\
\hline 109 & {$[350,375)$} & {$[84,97)$} & $\geq 5$ & D & 48.2 & 41.4 & 32.9 & 184 & 733 & 25.12 \\
\hline $110^{*}$ & {$[350,375)$} & {$[84,97)$} & $\geq 5$ & $E$ & 68.9 & 65.8 & 36.4 & 0 & 6451 & 0.00 \\
\hline 111 & {$[350,375)$} & {$[84,97)$} & $\geq 5$ & $\mathrm{~F}$ & 72.9 & 68.1 & 39.6 & 1902 & 19698 & 9.65 \\
\hline $112^{*}$ & {$[350,375)$} & {$[84,97)$} & $\geq 5$ & G & 68.9 & 65.8 & 36.4 & 0 & 3259 & 0.00 \\
\hline 113 & {$[350,375)$} & {$[84,97)$} & $\geq 5$ & $\mathrm{H}$ & 69.3 & 65.1 & 38.4 & 555 & 16925 & 3.28 \\
\hline $114 *$ & {$[375,400)$} & {$[84,97)$} & {$[0,5)$} & & 71.9 & 70.0 & 35.6 & 7 & 179 & 4.01 \\
\hline
\end{tabular}




\begin{tabular}{ccccccccccc}
115 & {$[375,400)$} & {$[84,97)$} & $\geq 5$ & $\mathrm{C}$ & 66.1 & 66.7 & 29.7 & 1988 & 28200 & 7.05 \\
116 & {$[375,400)$} & {$[84,97)$} & $\geq 5$ & $\mathrm{D}$ & 47.9 & 45.8 & 22.5 & 165 & 617 & 26.74 \\
$117^{*}$ & {$[375,400)$} & {$[84,97)$} & $\geq 5$ & $\mathrm{E}$ & 71.9 & 69.9 & 35.6 & 0 & 96 & 0.00 \\
118 & {$[375,400)$} & {$[84,97)$} & $\geq 5$ & $\mathrm{~F}$ & 81.4 & 80.3 & 39.4 & 1018 & 11487 & 8.86 \\
$119^{*}$ & {$[375,400)$} & {$[84,97)$} & $\geq 5$ & $\mathrm{G}$ & 71.9 & 69.9 & 35.6 & 0 & 1420 & 0.00 \\
120 & {$[375,400)$} & {$[84,97)$} & $\geq 5$ & $\mathrm{H}$ & 80.6 & 79.6 & 41.6 & 661 & 17462 & 3.79 \\
$121^{*}$ & {$[400,500)$} & {$[84,97)$} & {$[0,5)$} & & 70.5 & 66.9 & 38.3 & 2 & 1726 & 0.14 \\
122 & {$[400,500)$} & {$[84,97)$} & $\geq 5$ & $\mathrm{C}$ & 61.3 & 60.4 & 29.6 & 1779 & 73418 & 2.42 \\
123 & {$[400,500)$} & {$[84,97)$} & $\geq 5$ & $\mathrm{D}$ & 50.6 & 50.6 & 21.8 & 598 & 1350 & 44.25 \\
$124^{*}$ & {$[400,500)$} & {$[84,97)$} & $\geq 5$ & $\mathrm{E}$ & 70.5 & 66.9 & 38.3 & 0 & 40 & 0.00 \\
125 & {$[400,500)$} & {$[84,97)$} & $\geq 5$ & $\mathrm{~F}$ & 73.0 & 71.5 & 39.5 & 3691 & 23530 & 15.69 \\
$126^{*}$ & {$[400,500)$} & {$[84,97)$} & $\geq 5$ & $\mathrm{G}$ & 70.5 & 66.9 & 38.3 & 0 & 4129 & 0.00 \\
127 & {$[400,500)$} & {$[84,97)$} & $\geq 5$ & $\mathrm{H}$ & 79.1 & 82.0 & 42.5 & 2225 & 33796 & 6.58 \\
$128^{*}$ & $\geq 500$ & {$[84,97)$} & {$[0,5)$} & & 57.2 & 49.4 & 37.0 & 1 & 107 & 1.34 \\
$129^{*}$ & $\geq 500$ & {$[84,97)$} & $\geq 5$ & $\mathrm{~A}$ & 57.2 & 49.4 & 37.0 & 0 & $<1$ & 0.00 \\
$130^{*}$ & $\geq 500$ & {$[84,97)$} & $\geq 5$ & $\mathrm{~B}$ & 57.2 & 49.4 & 37.0 & 0 & $<1$ & 0.00 \\
$131^{*}$ & $\geq 500$ & {$[84,97)$} & $\geq 5$ & $\mathrm{C}$ & 57.2 & 49.4 & 37.0 & 0 & 56747 & 0.00 \\
$132^{*}$ & $\geq 500$ & {$[84,97)$} & $\geq 5$ & $\mathrm{D}$ & 57.2 & 49.4 & 37.0 & 0 & 9 & 0.00 \\
$133^{*}$ & $\geq 500$ & {$[84,97)$} & $\geq 5$ & $\mathrm{E}$ & 57.2 & 49.4 & 37.0 & 0 & 48 & 0.00 \\
134 & $\geq 500$ & {$[84,97)$} & $\geq 5$ & $\mathrm{~F}$ & 45.0 & 33.8 & 34.5 & 1827 & 26001 & 7.03 \\
$135^{*}$ & $\geq 500$ & {$[84,97)$} & $\geq 5$ & $\mathrm{G}$ & 57.2 & 49.4 & 37.0 & 0 & 6834 & 0.00 \\
136 & $\geq 500$ & {$[84,97)$} & $\geq 5$ & $\mathrm{H}$ & 70.0 & 74.1 & 35.2 & 1745 & 7913 & 22.06 \\
\hline & & & & & & & & & &
\end{tabular}

